\title{
STUDIES ON GASTRIC SECRETION
}

\author{
By THEODORE G. KLUMPP AND MORRIS A. BOWIE \\ (From the Medical Clinics of the Peter Bent Brigham Hospital, Boston, and the Lakeside \\ Hospital, Cleveland)
}

(Received for publication June 30, 1932)

In the voluminous literature on gastric function one traces the gradual destruction of the hope that gastric analysis can give exact diagnostic information. The literature reveals, instead, the enormous variability of functional activity of the normal stomach. Achlorhydria has been observed frequently in persons who continue to be healthy and otherwise entirely normal. In pernicious anemia achlorhydria is not always present, as the findings of Castle (1) and his associates indicate. When so-called hyperacidity is found, it is often regarded as the cause of certain symptoms, but is as often present without symptoms. Despite the fact that we cannot ordinarily establish a definite diagnosis on the basis of gastric analysis alone, it gives supportive information of unquestioned value.

The fashion in test meals has, over a period of years, favored foods common to the usual dietary. The idea has been that bread and water, milk, broth or even a full meal offered a physiological stimulus, and such meals as the Ewald, Boas, Sahli and Riegel have enjoyed the favor of many years. In recent years a dilute solution of alcohol has gained vogue as a test substance because it is a clear solution and provokes a significant secretion of gastric juice. In 1920, the action of histamine on gastric secretion was first studied by Popielski (2). In 1922 stimulation of gastric secretion by histamine was applied to man by Carnot, Koskowski and Libert (3). Since then it has been increasingly used in gastric analysis, largely because it was thought to give a more intense, possibly maximum stimulation.

At the present time, the Ewald, alcohol, and histamine stimuli are widely used in this country. They have been extensively studied, but these studies have yielded very little information by means of which their actions can be adequately compared. This is due to the fact that insufficient allowance has been made for the wide range of functional variation of normal gastric secretion. In order to balance this variable in considering two test meals, it is necessary to give both of them to the same subject, under standard conditions, and compare adequate series of such duplicate analyses. With this in mind, a study of gastric function was undertaken. A total of three hundred and fifty-seven analyses were made on ninety-eight subjects under controlled conditions. 
METHODS

A uniform technique was adopted. The stomach was drained of its fasting contents. After the test substance had been given, samples of gastric contents were withdrawn at fifteen minute intervals for one hour. In the case of histamine, the stomach was drained at each aspiration. With the Ewald and alcohol meals, the contents were aspirated to measure the volume, and the surplus over $10 \mathrm{cc}$. reinjected. At the end of the hour the stomach was emptied. After a rest of fifteen minutes the second test meal was administered and specimens collected as before. At time intervals that varied from several days to weeks, the analyses were duplicated, using a different combination of the Ewald, histamine and alcohol stimuli. Most of the subjects were repeatedly examined. As many as twenty-four complete gastric analyses were obtained from a given individual. The Ewald meal consisted of two slices of white bread and $200 \mathrm{cc}$. of water. Of alcohol $50 \mathrm{cc}$. of a 7 per cent solution were given. Either $0.5 \mathrm{mgm}$. or $1 \mathrm{mgm}$. of histamine sulphate was injected subcutaneously.

\section{EXPERIMENTAL}

\section{Individual variations in gastric secretion}

Bloomfield and Keefer (4) found on re-examination of the same person that acidity and volume of gastric secretion remained practically constant. Our findings in multiple analyses spread over varying intervals of time, suggest that in general the acidity values fall within a broad range. There were, however, occasional disturbing fluctuations. Thus, subject A. P. attained a total acidity of 71 units of tenth normal sodium hydroxide on a fractional gastric analysis after histamine. Three weeks later the level of all fractions was entirely out of the previous range and he reached the extraordinary peak of 149 units of total acid after histamine. Another subject, D. H., in one gastric analysis had achlorhydria in two of four fractions after histamine, whereas in another gastric analysis he had 70 units of free acid. A third subject, who had had eight complete examinations, in three secreted no free hydrochloric acid after histamine and alcohol, whereas in three there were between 10 and 36 units of free acid in all fractions. In the remaining two gastric analyses the free acid was low. These are not isolated occurrences, but serve to illustrate the occasional fluctuations encountered, as well as to point out the danger of making a diagnosis of achlorhydria on a single aspiration of gastric contents or even a single gastric analysis. Henning (5) in 1928 emphasized the necessity of conducting several histamine tests before concluding that the gastric cells have lost the power to secrete. Best and McHenry (6) conclude: "One negative histamine test is therefore not conclusive evidence of achlorhydria."

The volume of gastric secretion on repeated analyses proved extremely variable; and no relation was found between volume of juice secreted and 
acidity, which is in accord with the findings of Bloomfield and Keefer (4) and Comfort and Osterberg (7). The latter authors doubt the diagnostic value of volume estimation.

\section{Comparison of Ewald and alcohol test meals, and histamine}

The extensive use and study of these test substances has provided very little information by means of which their powers to stimulate the flow of gastric juice could be accurately compared. In an attempt to do this, three series of duplicate analyses were made, using respectively the combinations: alcohol and histamine, Ewald and histamine, and alcohol and Ewald. The arithmetical averages are given in Table I. The coefficient of dispersion ( $\left.\frac{\text { average deviation }}{\text { mean }}\right)$ is used as the index of reliability. The particular suitability of the index for the type of comparisons drawn in this study has been pointed out by Karl Pearson (21). The coefficient of dispersion was not calculated for volumes of secretion throughout, because these values showed no consistent trend nor was it calculated for the two cases in which gastric analysis was done following injection of calcium chloride.

Histamine and alcohol. In the first series, twenty-seven subjects received both the alcohol meal and the histamine meal. In all, sixty-one analyses were made under the standard conditions described previously. Following histamine, twenty-nine fractions contained no free hydrochloric acid, whereas after alcohol thirty-nine fractions contained no free acid. From the figures of Table I it will be noted that histamine elicits consistently higher values for both free and total acidities. After histamine the acidity reaches a maximum at the end of thirty minutes, falling off thereafter. The rise in acidity after alcohol is more gradual and reaches a maximum at the end of an hour. The volume of secretion is practically the same in both instances, although it is to be remembered that the alcohol test starts off with a gratuity of $50 \mathrm{cc}$.

Histamine and Ewald. Nineteen subjects were examined. A total of forty-five gastric analyses were made. Seventeen specimens contained no free hydrochloric acid after the bread and water meal, whereas after histamine the number without free acid was fifteen. From Table I the following facts are evident: The free acid after the Ewald test is significantly lower than after histamine. The total acidity after histamine reaches a maximum at the end of forty-five minutes, and exceeds that of the highest Ewald value by a small margin. Both free and total Ewald acidities reach a maximum at the end of an hour. Molinari-Tosatti (8) found that the secretion after a similar test meal lasted longer than after histamine. The volume of gastric contents is slightly higher after the bread meal. The initial Ewald intake of $200 \mathrm{cc}$. of water must be taken into consideration in drawing comparisons. 
GASTRIC SECRETION

\begin{tabular}{|c|c|c|c|c|c|c|c|}
\hline & 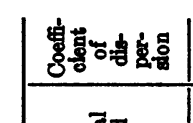 & | $\begin{array}{l}\text { ir } \\
\text { : }\end{array}$ & \multicolumn{2}{|c|}{ 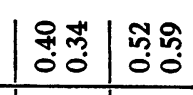 } & $\mid \begin{array}{l}3 \\
\text { 9. } \\
0\end{array}$ & \multicolumn{2}{|r|}{ 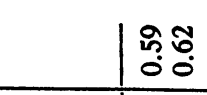 } \\
\hline & 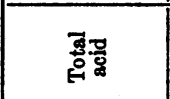 & 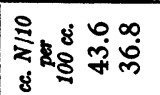 & 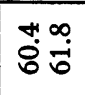 & 華号 & 急号 & & 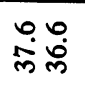 \\
\hline & 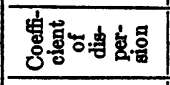 & 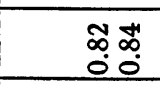 & ț: & 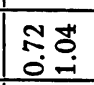 & $\begin{array}{ll}0 \\
0 \\
0.00\end{array}$ & & 赵 \\
\hline & $\frac{8}{20}$ & 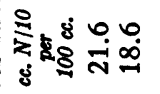 & 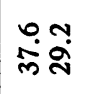 & $\mid$ & कें & & โุ \\
\hline & 嵩眘 & $8: 0 \%$ & कृ & 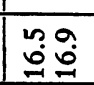 & 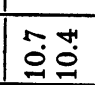 & & 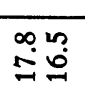 \\
\hline \multirow{5}{*}{ 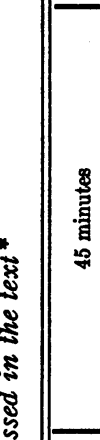 } & 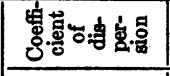 & 공 है & $\begin{array}{l}\text { 앙 } \\
\text { S. }\end{array}$ & 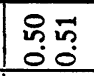 & 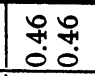 & 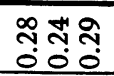 & तु? \\
\hline & 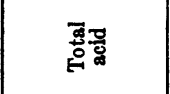 & 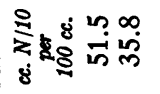 & 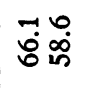 & 孚承 & $\mid \begin{array}{l}a m \\
\text { ar } \\
\text { in in }\end{array}$ & 궁ำ & 过皿 \\
\hline & 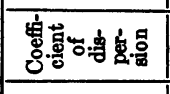 & 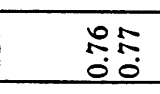 & 중영 & $\begin{array}{ll}0 \\
0 \\
0\end{array}$ & \begin{tabular}{|l|l}
170 \\
80 \\
\end{tabular} & 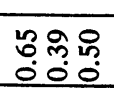 & 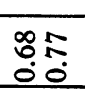 \\
\hline & 뭄 & $\begin{array}{l}8 \\
\bar{n} \\
8\end{array}$ & 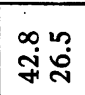 & 东我 & Fi & 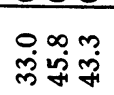 & พิ่ \\
\hline & 㝒眘 & 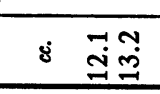 & 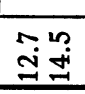 & Pid & 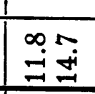 & 업표 & 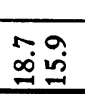 \\
\hline \multirow{5}{*}{ 咅 } & 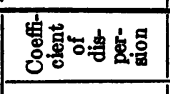 & 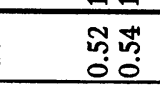 & $\begin{array}{l}0.7 \\
0 \\
0 \\
0\end{array}$ & 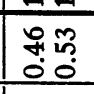 & $\begin{array}{l}0+\infty \\
9 \\
0 \\
0\end{array}$ & 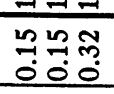 & 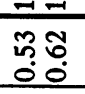 \\
\hline & 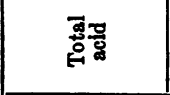 & 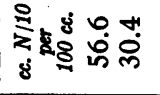 & 엉혀 & 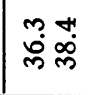 & 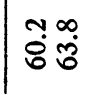 & 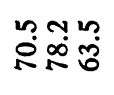 & $\dot{m}$ \\
\hline & 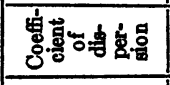 & $\begin{array}{l}0 \\
\substack{0 \\
0 \\
0} \\
0\end{array}$ & 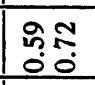 & R8 & 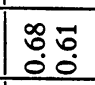 & 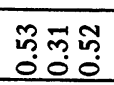 & 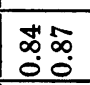 \\
\hline & 缕票 & 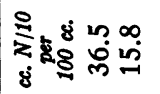 & స్లి & 足足 & 若年 & 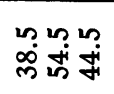 & 可出 \\
\hline & 官首 & 8 تేْ & $\overrightarrow{\mathrm{s}}$ & 式草 & 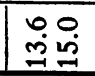 & 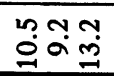 & 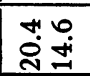 \\
\hline \multirow{5}{*}{ 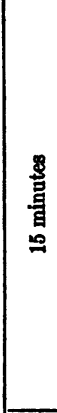 } & 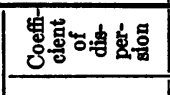 & 象: & 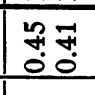 & 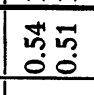 & 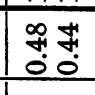 & $\begin{array}{l}5 \pi \\
500 \\
0\end{array}$ & 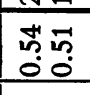 \\
\hline & 娶: & 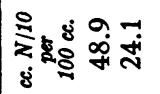 & ํํำ & 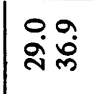 & 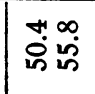 & 象是:? & फु- \\
\hline & 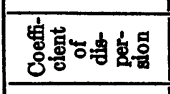 & 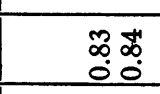 & 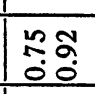 & 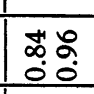 & 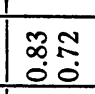 & 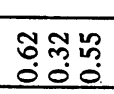 & åa \\
\hline & 悉兽 & 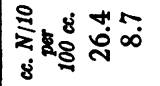 & 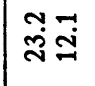 & 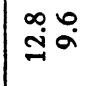 & 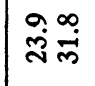 & 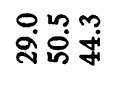 & 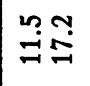 \\
\hline & 它首 & $8 \quad 8 \overrightarrow{0}$ & 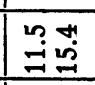 & 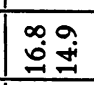 & 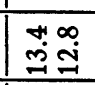 & تุon & 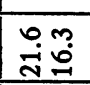 \\
\hline & 䚁晏曼 & $\hat{A N}$ & 92 & Aュ & 77 & 000 & นูก \\
\hline & 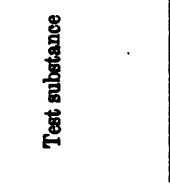 & 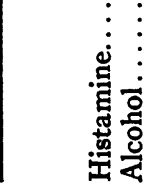 & 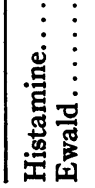 & 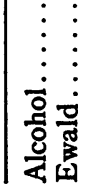 & 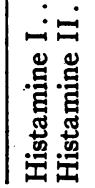 & 递 & 通 \\
\hline
\end{tabular}


THEODORE G. KLUMPP AND MORRIS A. BOWIE

\begin{tabular}{|c|c|c|c|c|c|c|}
\hline 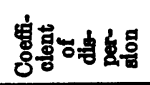 & స్తి & 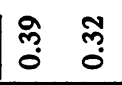 & |ู่: & & & \\
\hline 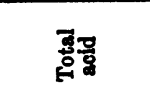 & 言 & 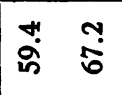 & $\hat{i}$ & ํํํํำ & 递家 & \\
\hline 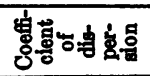 & 总: & भी दु & $\left.\right|_{0} ^{\infty} 0$ & & & \\
\hline 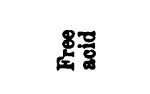 & 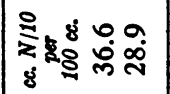 & 品 & 芦 & 임ㅇㅎㅁ & ㅇㅇㅇ & \\
\hline 高首 & 8 ชิ & $\stackrel{m}{=} \vec{a}$ & $\vec{a} \quad \stackrel{\infty}{0}$ & 웅 & 잉. & \\
\hline 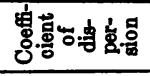 & 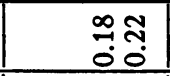 & $\begin{array}{cc}0 \\
0 \\
0 \\
0\end{array}$ & $\begin{array}{|ll|}8 & 9 \\
0 & 0 \\
\end{array}$ & & & \\
\hline 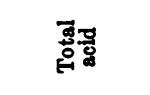 & 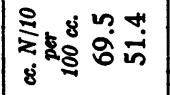 & :ี : : & 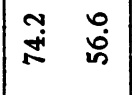 & 옴ํำ & 얼워 & \\
\hline 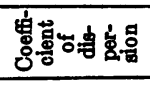 & 兽 & $\begin{array}{ll}3 \\
3 \\
0\end{array}$ & $\begin{array}{|cc|}0 & 0 \\
0 & 0 \\
0\end{array}$ & & & \\
\hline 8 & 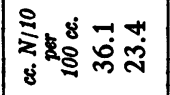 & $\stackrel{+}{+}$ & 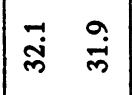 & 일류 & :어ํ & \\
\hline 官首 & 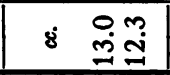 & $\stackrel{\infty}{\circ} \stackrel{\infty}{\circ}$ & $\begin{array}{|ll|} & \infty \\
\infty & \infty \\
\end{array}$ & 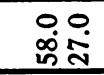 & ن욜요 & \\
\hline 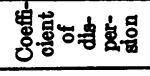 & 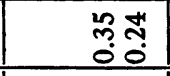 & $\begin{array}{cc}0 \\
0 \\
0 \\
0\end{array}$ & 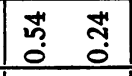 & & & \\
\hline 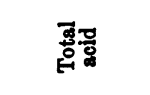 & 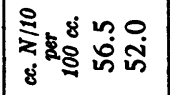 & i̊ & $\vec{j}$ & $\dot{\dot{q}}$ & ن요 & \\
\hline 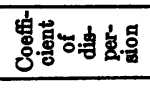 & 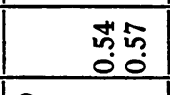 & $\begin{array}{ll}\infty \\
0 \\
0\end{array}$ & $\begin{array}{ll}0 & 0 \\
0 & 0 \\
\end{array}$ & & & \\
\hline $8 \%$ & 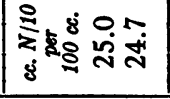 & 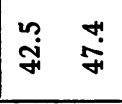 & 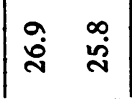 & 링요 & 요료 & \\
\hline 宫首 & 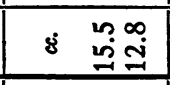 & $\stackrel{\widetilde{O}}{\mathrm{I}}$ & $\begin{array}{|cc|} & \infty \\
\varrho & \stackrel{9}{9} \\
\end{array}$ & 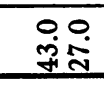 & ن욤 & \\
\hline 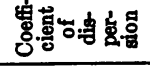 & ర్లోల్లో & $\begin{array}{c}\tilde{m} \\
0 \\
0\end{array}$ & 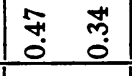 & & & \\
\hline 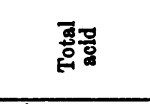 & 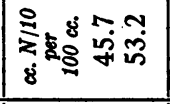 & $\hat{\dot{B}} \overline{\dot{0}}$ & 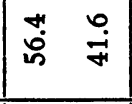 & 我足 & 웅용 & \\
\hline 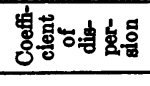 & 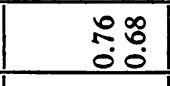 & 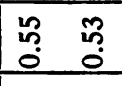 & $\begin{array}{|cc|}a & 0 \\
0 & 0 \\
0\end{array}$ & & & \\
\hline 800 & $\begin{array}{llll}0 & 0 & 0 \\
3 \\
3\end{array}$ & 芦 芦 & 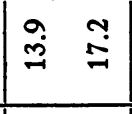 & 递家 & 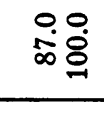 & 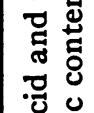 \\
\hline 富目 & 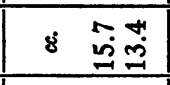 & $\stackrel{m}{\stackrel{0}{0}}$ & $\begin{array}{ll}m \\
0\end{array}$ & 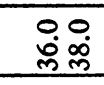 & 就 & \\
\hline 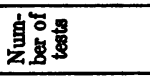 & $\approx \approx$ & $\Rightarrow=$ & $\begin{array}{ll}a & a\end{array}$ & -1 & -1 & \\
\hline $\begin{array}{l}\text { : } \\
\text { 害 } \\
\text { 意 }\end{array}$ & 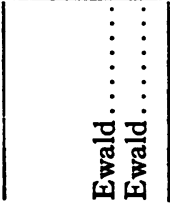 & 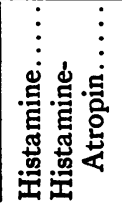 & 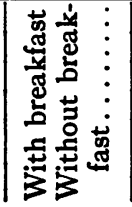 & 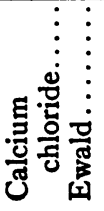 & 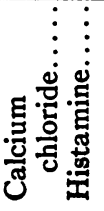 & 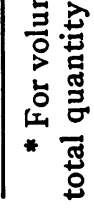 \\
\hline
\end{tabular}


Alcohol and Ewald. Thirty-four analyses were made using seventeen subjects. After the Ewald test twenty-eight fractional tests contained no free acid, whereas after alcohol only eighteen samples were negative for free acid. A study of Table I shows that under similar conditions the Ewald meal gives higher values for total acidity than the alcohol test. This is in accord with the previous findings in which the histamine values were more nearly approached by the Ewald test than by the alcohol test. The free acid values after the Ewald meal are again low. The volumes of secretion show no significant trend.

\section{Effects of repeated and prolonged stimulation of gastric secretion}

In order to study the changes taking place in gastric secretion during repeated or prolonged stimulation, the following experiments were performed: The subject was given one of the test substances and four aspirations were made, in the usual manner, at intervals of fifteen minutes. Care was taken to drain completely the stomach with the last aspiration. After a rest of fifteen minutes the same test substance was then repeated and a second gastric analysis performed. A series of patients was studied by means of each of the three test stimulants: alcohol, Ewald meal, and histamine. In order to study further the secretory function during prolonged stimulation, three successive administrations of histamine were given at intervals of forty-five minutes, and the fractional samples of the analyses compared in a series of patients.

We were interested in discovering: (a) Whether repeated stimuli brought forth the same or a lessened response, in other words whether the phenomenon of fatigue could be demonstrated; $(b)$ Whether there were any differences in the capacities of the test substances to elicit a renewed or prolonged secretion; $(c)$ Whether the so called "maximal" stimulus of histamine left in its wake a pronounced fatigue or a refractive period; (d) Whether the Ewald meal, a so called "physiological" stimulus, evoked a pattern of response different from that caused by histamine and alcohol.

Ewald tests. Two successive Ewald meals were given as described above, to twelve subjects. The averages represented in Table I show that the first test meal evokes the progressive increase in acidity noted previously. In the first fraction after the second test meal the acidity is greater than in the corresponding fraction after the first test meal. In the remaining fractions after the second test meal there is both an actual and comparative falling off of acidity. In other words, after the second test meal there is a short interval of augmented acid output followed by a lessened ability to respond.

Alcohol tests. Two successive alcohol tests, as described, were given to twenty-five subjects. A total of twenty-eight duplicate tests were made. It will be noted from Table I that the maximum response appears 
in the third specimen after the first test. After the second administration of alcohol the acidity in the first two fractions is greater than in the corresponding fractions of the initial analysis. In the remaining two fractions the acidity diminishes absolutely and relatively. The curve is similar to that obtained from the Ewald meals, except that after alcohol the accumulative effect is slightly prolonged.

Histamine tests. Two successive gastric analyses were made after histamine, as in the manner previously described, on forty-one subjects who received in all forty-six paired gastric analyses. Examination of Table I shows that the average free and total acidity becomes maximum in the second specimen (one-half hour after injection) in both tests. The first two specimens of the second test are more acid than the corresponding fractions of the first test. In addition it is noted that the peak of both analyses is attained in the second specimen of the second test. The last two specimens are lower than the homologous samples of the first test. In other words two successive injections of histamine give a cumulative effect, followed by a decline in secretory activity.

In order to extend the observation described above, and to determine whether the cumulative effect is carried to a third stimulation, six subjects were given three successive injections of histamine at intervals of three quarters of an hour, fractions being collected every fifteen minutes as before. The peak of acidity (Table I), after the first injection came, as before, in the half hour (second) specimen. After the second injection of histamine the output of acid was augmented in all three specimens. The maximum was attained in the second specimen followed by a definite falling off in the third specimen, which was, nevertheless, higher than the corresponding third specimen after the first injection. After the third injection of histamine there was slight augmentation of acidity in the first specimen, but a drop thereafter.

These experiments demonstrate that repeated stimuli increase the secretion of acid. This holds for the Ewald and alcohol meals, as well as for histamine. This does not seem to be a true arithmetical summation phenomenon. When three successive stimuli are given, the augmentation of acidity after the third is less than after the second. Following the initial increase in output of acid on repeated stimulation, there is a definite reduction suggestive of a fatigue phenomenon. Ivy and Javiois (9) and Lim (10) observed a diminution of response on successive administration of histamine in dogs, although the interval between injections ( 3 hours) was probably too long to provoke any additive effect. Best and McHenry (6) state, "The gastric secretory mechanism affected by histamine is not easily completely fatigued, but the effects of an injection of the amine is greatly reduced by the recent previous administration of the substance." Polland (11), on the other hand, found that repeated 
injections of histamine produced the same volume and acidity. Further investigations of the laws governing gastric secretion would be desirable.

\section{The effect of atropine after histamine stimulation}

Polland (11), found that atropine, after histamine, caused a marked decrease in volume, a rise in titrable acidity, and a fall in total acid in the gastric secretions. Best and McHenry (6), state; "There is fairly general agreement that atropine does not prevent the action of histamine on the human gastric glands." Using eleven subjects, we have made similar studies. After the administration of 1 milligram of histamine sulphate aspirations of four samples were made at 15 minute intervals. After a rest of 15 minutes, 1 milligram of histamine sulphate and from gr. 1/100 to gr. 1/50 of atropine sulphate were given in the same syringe. Four aspirations were made as before. The results are represented in Table I. The values for volume showed, as before, no definite trend. The second injection gave in the first two specimens an augmentation of titrable acidity comparable to that which might have been expected if histamine alone had been given. The titrable acidity of the third and fourth specimens, however, either equaled or exceeded that of the corresponding specimens of the first analysis. This means that the addition of atropine prolonged the high level of titrable acidity, inhibiting the falling off which generally occurs in the last two specimens. To this extent atropine augmented the titrable acidity as Polland had noted.

\section{$V$. Influence of breakfast on gastric analysis}

Inasmuch as patients occasionally misunderstand instruction and come to the clinic after they have had breakfast, it was thought desirable to study a group of nine subjects making duplicate gastric analyses, with and without breakfast. It is interesting that some of the test meals recommended, such as the Riegel and Fischer meals, constitute practically a full meal. It is admitted, of course, that the presence of the breakfast introduces an unknown variable, which, as a general rule, makes study of the gastric contents unsatisfactory. To what extent a previous breakfast interferes with the proper interpretation of gastric analyses we were interested in knowing. On one day the subject was examined, after having eaten a customary breakfast at the usual time. The gastric contents were aspirated and the analyses, thereafter, conducted in the manner previously described, using one of the test substances. On another day, the patient came in without breakfast, and the analyses were repeated using the same test substance. The results given in Table I show that the volumes of secretion were in close agreement. The free acid shows no constant trend, although, on the whole, it was slightly lower after a previous breakfast, due perhaps to the buffer action of the food. After a previous breakfast the total acidity showed a distinctly 
higher level in all samples. This might possibly be due to the augmentative effect of repeated stimulation which has been pointed out above.

Free acid values, then, are slightly lower and total acidities markedly higher after the subject has had a previous breakfast than when he is examined in the customary fasting condition.

\section{Effect of intravenous calcium chloride on gastric secretion}

The recent interest in the use of intravenous calcium chloride to relieve smooth muscle spasm prompted us to investigate its effect on gastric secretion. Two patients were given $10 \mathrm{cc}$. of 5 per cent calcium chloride intravenously after which fractional samples of gastric secretion were removed every fifteen minutes for one hour. The volume and acidity were then compared, in one case, with an ordinary histamine analysis, and in the other with an Ewald test.

The results in Table I indicate that intravenous calcium chloride is followed by an increased gastric secretion which, in the two subjects studied, was comparable in volume and acidity to that produced in Ewald and histamine tests.

\section{COMMENT}

The comparative merits of the various test meals and substances has been the subject of wide discussion. The Ewald meal has been criticized because of the low acid values or even achlorhydria which it evokes in the normal stomach. In this respect the present study shows that the alcohol test is no marked improvement. The histamine test has been objected to on the ground that it introduces an unphysiological and unnatural stimulus. In this connection, it is interesting that Ivy (12) has recently isolated from the gastric mucosa histamine sulphate which he identifies with gastrin. In considering the virtues of the various test substances for gastric analysis, almost no regard has been given to the influence of the test substances on duodenal regurgitation and the rate of emptying of the stomach. Neale and Klumpp (13) have shown that during the height of histamine activity gastric contents do not appear in the duodenum and pancreatic secretion into the duodenum is inhibited. Lim, Matheson, and Schlapp (14), Osborne (15) and others agree that pancreatic secretion is decreased during histamine activity. This explains why gastric aspirations after histamine are uniformly clear, colorless, and unstained with bile. With the other test substances the ebb and flow through the pylorus introduces an uncontrolled variable which modifies both volume and acidity.

The stomach shares with the kidneys, heart, lungs and other organs a wide range of functional capacity and a large margin of reserve. Because of this, a mild test stimulus, comparable with the usual physiological functional demand, is unreliable because the reserve ability of the organ 
will mask early functional impairment. In order to detect such slight or early impairment, it is necessary to apply a maximal stimulus, a functional load which will try the organ to its full capacity. It is our belief that this principle, which has been recognized and applied in the study of renal, pulmonary, pancreatic and cardiac disorders, should be considered in studying gastric function. A stimulating agent should be used which is sufficiently intense to overcome the large margin of functional reserve that may mask the defect. This concept has been previously expressed and advantageously applied in the study of disease by Bloomfield and Polland (16). It has been amply demonstrated that histamine provides a more intense stimulus than the other test substances; in adequate dosage, possibly a maximal stimulus. This we believe is the most satisfactory single agent for the study of gastric function.

The advantages of the histamine stimulus over the various test meals have been recognized by Bloomfield and Polland (16), Rudd (17), Carnot and Libert (18), Andresen (19) and others. The following is a summary of some of these advantages.

1. The variables of salivary dilution, appetite or anorexia, and rate of eating incidental to test meals are eliminated (15). 2. The gastric contents are uniformly clear, colorless and watery. 3. A tube of small bore may, therefore, be used, making nasal passage possible, especially for children and uncooperative patients. Such a tube and tip has been described by one of us (20). The stomach may be emptied at each fractional aspiration. 5. The peak of secretory stimulation is attained within forty-five minutes, thus making the test shorter than when the Ewald and alcohol meals are used. 6. The masking buffer action and reaction of the meal is not present. 7. Histamine provides a strong stimulus. The advantages of the functional load in gastric analyses has been discussed. 8. The histamine test gives more nearly constant results on repeated examinations of the same individuals (15). 9. The histamine test is the only one that can be relied on for the detection of achlorhydria. 10. During the height of histamine response neither duodenal regurgitation nor loss of gastric contents occurs (12).

\section{SUMMARY}

1. In a given individual the secretion of acid in response to a uniform stimulus tends in general to remain within a broad range. Occasional wide fluctuations are, however, encountered.

2. One negative histamine test is not conclusive evidence of achlorhydria.

3. The volume of gastric secretion in response to a uniform stimulus fluctuates widely, and no relation between volume and acidity is found.

4. In a series of duplicate analyses histamine elicited consistently higher values for free and total acid than the Ewald and alcohol tests. 
The maximum secretion of acid was attained between 30 and 45 minutes after the injection of histamine. After the Ewald and alcohol tests the maximum response came at the end of an hour or later.

5. The alcohol test gave higher values for free acid than the Ewald meal, but the latter evoked higher total acidities in duplicate analyses.

6. In duplicate analyses, achlorhydria appeared more frequently after the Ewald meal than after alcohol, and least frequently after histamine.

7. Administration of repeated gastric stimuli induced a temporary augmentation of acidity after each, followed by a pronounced falling off, suggestive of a fatigue phenomenon. There was no fundamental difference in the power of the three test meals to elicit this response.

8. Atropine in moderate doses tends to prolong the high level of titrable acidity which ordinarily occurs after histamine.

9. A previous breakfast tends to cause lower acid values and higher total acidities in response to a standard stimulus.

10. Intravenous calcium chloride increases gastric secretion.

11. The theoretical and practical advantages of studying gastric function by means of the histamine stimulus are discussed.

The authors are happy to acknowledge the generous cooperation of Dr. A. J. Beams.

\section{BIBLIOGRAPHY}

1. Castle, W. B., Heath, Clark, W., and Straus, M. B., Am. J. M. Sc., 1931, clxxii, 741. Observations on the Etiologic Relationship of Achylia Gastrica to Pernicious Anemia. IV. A Biologic Assay of the Gastric Secretion of Patients with Pernicious Anemia Having Free Hydrochloric Acid and that of Patients without Anemia Having no Free Hydrochloric Acid and of the Rôle of Intestinal Impermeability to Hematopoietic Substances in Pernicious Anemia.

2. Popielski, L., Arch. f. d. ges. Physiol., 1920, clxxviii, 214. B-imidazolyläthylamin als mächtiger Erreger der Magendrüsen.

3. Carnot, P., Koskowski, W., and Libert, E., Compt. rend. Soc. de biol., 1922, lxxxvi, 575. L'Influence de l'histamine sur la Sécrétion des sucs digestifs chez l'Homme.

4. Bloomfield, A. L., and Keefer, C. S., Arch. Int. Med., 1926, xxxviii, 145. Clinical Physiology of the Stomach. Simultaneous Quantitative Observations on Gastric Secretory Volume, Acidity and Motility.

5. Henning, N., Munchen. med. Wchnschr., 1928, lxxv, 1752. Histaminprobe und Prognostik der Achylien.

6. Best, C. H., and McHenry, E. W., Physiol. Rev., 1931, xi, 371. Histamine.

7. Comfort, M. W., and Osterberg, A. E., J. A. M. A., 1931, xcvii, 1141. Gastric Secretion after Stimulation with Histamine in the Presence of Various Types of Gastric and Duodenal Lesions.

8. Molinari-Tosatti, P., Arch. di sc. biol., 1929, xiii, 97. Secrezione gastrica da istamina e secrezione da pasto.

9. Ivy, A. C., and Javois, A. J., Am. J. Physiol., 1925, 1xxi, 604. Contributions to the Physiology of Gastric Secretion, VI. The Stimulation of Gastric Secretion by Amines and Other Substances. 
10. Lim, R. K. S., Am. J. Physiol., 1924, lxix, 318. On the Relationship between the Gastric Acid Response and the Basal Secretion of the Stomach.

11. Polland, W. S., J. Clin. Invest., 1930, ix, 319. The Effect of Atropine upon Gastric Secretion after Histamine Stimulation.

12. Ivy, A. C., J. A. M. A., 1931, xcvii, 1141. Abstract of Discussion of paper by Comfort, M. W., and Osterberg, A. E. (7).

13. Neale, A. V., and Klumpp, T. G., J. Clin. Invest., 1930, ix, 197. The Action of Histamine on the Pancreas.

14. Lim, R. K. S., Matheson, A. R., and Schlapp, W., Quart. J. Exper. Physiol., 1923, xiii, 333. An Improved Method for Investigating the Secretory Function of the Stomach and Duodenum in the Human Subject.

15. Osborne, W. A., Australian J. Exper. Biol. and M. Sc., 1928, v, 171. Low Blood Pressure and Secretory Activity.

16. Bloomfield, A. L., and Polland, W. S., J. A. M. A., 1929, xcii, 1508. The Diagnostic Value of Studies of Gastric Secretion.

17. Rudd, G. V., Brit. J. Exper. Path., 1931, xii, 226. Observations on the Acidity and Chloride Content of the Gastric Juice. II.

18. Carnot, P., and Libert, E., La Méd., 1925, vi, 757. Les Repas d'Épreuve et le Test de l'Histamine comme Mesure de l'Activité Sécrétoire de l'Estomac.

19. Andresen, A. F. R., J. A. M. A., 1931, xcvii, 1141. Abstract of Discussion of paper by Comfort, M. W., and Osterberg, A. E. (7).

20. Klumpp, T. G., Am. J. Dis. Child., 1931, xli, 360. A Tip for the Gastroduodenal Tube Designed for Nasal Passage in Infants and in Children.

21. Pearson, Karl, Phil. Tr. Roy. Soc., Series A, 1896, clxxxvii, 276. Regression, Heredity and Panmixia. 